\title{
An Intelligent System to Avoid Collision in Vehicle based on Can Protocol
}

\author{
Ojas V. Barve \\ Dept. of Electronics \& \\ Telecommunication \\ Engineering \\ D. J. Sanghvi COE \\ Mumbai, India.
}

\author{
Neeraj Ramkumar \\ Dept. of Electronics \& \\ Telecommunication \\ Engineering \\ D. J. Sanghvi COE \\ Mumbai, India.
}

\author{
Revathi A. S. \\ Dept. of Electronics \& \\ Telecommunication \\ Engineering \\ D. J. Sanghvi COE \\ Mumbai, India.
}

\begin{abstract}
Driver and passenger safety are one of the prime concerns in a modern day vehicle. Alarming statistics of accidents and increased number of vehicles on road demands for an intelligent safety mechanism that helps the driver in handling immediate precarious situations like sudden probability of a collision. The work proposes a mechanism that not only computes the deceleration of vehicle due to braking and displays the braking intensity through an array of LED but also involves an emergency braking system and communicate it to the vehicles that are following it in lambertian [1] range of IR transmitter module to avoid any collision pre-hand, due to any situation that may arise and cause immediate deceleration of the vehicle ahead. An Arduino UNO microcontroller will be interfaced with an accelerometer and ultrasonic sensor that senses the deceleration levels, a LED array to display the braking intensity and an IR transceiver module for inter-vehicle communication that would transmit pulses whose frequency is modulated proportional to the braking intensity level. To take decision and automatically control the motion of the following vehicle, a collision avoidance system consisting of Arduino UNO microcontroller is implemented that actuates the brake control system in case of drastic situations. All the communications between different systems will be done through the common CAN Bus which will help us incorporate this system into automobiles thereby reducing time to market.
\end{abstract}

\section{Keywords}

Keywords are your own designated keywords which can be used for easy location of the manuscript using any search engines.

\section{INTRODUCTION}

As per a report submitted by National Institute of Disaster Management, in India every 80 seconds there is a road accident i.e. 1080 accidents per day[2]. Human errors amount to $93 \%$ of all accidents and according to police, rear-end collision constitute $30 \%$ of all fatal accidents An accident in which the rear of a vehicle is hit by a trailing car, due to immediate change in car's speed as a result of emergency or hard brake applied is termed as rear end collision. The most common rear-end collision is a collision where a car's rear is rammed by a heavy truck. Also more collisions that involve rear end crash do occur in broad daylight with good visibility conditions on a straight road and in good weather condition but nonetheless bad weather or bad visibility could also lead to rear end collision. Reducing traffic accidents is one of the main objectives of any transportation system. Accidents due to rear-end collisions very common and it does become one of the research soughed topic in the automotive sector. As the
Driver's ability to keep attention has a key role to play in prevention of such collisions, an automated system that assists the driver would prove to be of great help in reducing these accidents significantly.

\section{TECHNOLOGY USED}

Controller Area Network or CAN protocol is a method of communication between various electronic devices like engine management systems, active suspension, ABS, gear control, lighting control, air conditioning, airbags, central locking etc. embedded in an automobile. An idea initiated by Robert Bosch $\mathrm{GmbH}$ in 1983 to improve the quality of automobiles thereby making them more reliable, safe and fuel efficient. With the developments taking place in the electronics and semiconductor industry the mechanical systems in an automobile were being replaced by more robust electronics system which had an improved performance. New technologies, products and inventions with added or improved functions started to shape a complete new era for the automobile industry which promised more robust vehicles with use of electronics. The increasing number of electronic devices used communication signals with more complex interrelations between them. Thereby making the life difficult for automobile engineers when they designed systems wherein one electronic device needs to communicate with others to operate. Realizing the problem of communication between different electronic modules Robert Bosch came up with this new protocol called CAN which was first released in 1986. CAN provide a mechanism which is incorporated in the hardware and the software by which different electronic modules can communicate with each other using a common cable [11] [12]

\section{PROPOSED SYSTEM}

\subsection{Components required}

1) Accelerometer:-By measuring the amount of static acceleration due to gravity, one can find out the angle the device is tilted at with respect to the earth. By sensing the amount of dynamic acceleration, one can analyse the way the device is moving. Measuring tilt and acceleration parameters are integral to understand the vehicle dynamics of a car. By quantifying the lateral and longitudinal acceleration, it is possible to estimate if the car is undergoing hard braking. This makes it an integral sensor in any type of collision avoidance system. In a similar fashion, High $\mathrm{G}$ force accelerometers are the industry standard way of detecting car crashes and deploying airbags at just the right time and for testing. For the proposed system, adxl335 accelerometer sensor which has a range of $+/-3 g$ is 
used which is more than sufficient for our needs of prototyping and concept validation. [9] [4]

2) Ultrasonic sensors: They are based on measuring the properties of sound waves with frequency above the human audible range. They are based on three physical principles: time of flight, the Doppler effect, and the attenuation of sound waves. Ultrasonic sensors are non-intrusive in that they do not require physical contact with their target, and can detect certain clear or shiny targets otherwise obscured to some vision-based sensors. For this project, HC-SR04 Ultrasonic Sensor is used . [4].

3) MCP2515 and MCP2551: The MCP2551 is a highspeed CAN transceiver, fault-tolerant device that serves as the interface between a CAN protocol controller and the physical bus. The MCP2551 provides differential transmit and receive capability for the CAN protocol controller and is fully compatible with the ISO-11898 standard, including $24 \mathrm{~V}$ requirements. It will operate at speeds of up to $1 \mathrm{Mb} / \mathrm{s}$. Microchip's MCP2515 is a standalone CAN controller which implements CAN 2.0B. It is capable of transmitting both standard frames, extended frames and remote frames. It has 2 acceptance masks and 6 acceptance filters that are used to filter out unwanted messages. It interfaces with microcontrollers using an industry standard Serial Peripheral Interface (SPI).

4) Actuators: Actuators are needed to automatically engage brakes to avoid collision. Many different methods exist for actuating the brakes. Each method has different applications and advantages disadvantages. The most common methods currently found include pneumatic, direct electronic, and electro-mechanical. Considering ease of use and packaging constraints in existing systems, an electronic system with the help of servo motors has been proposed in the system.

5) IR Receiver: An IR receiver is a photo diode that generates electrical signal proportional to the intensity of IR rays incident on it. The signal is demodulated to receive the information signal. This information is the input to the collision avoidance system to start with control algorithm.

\subsection{System Functioning and Block Diagram}

The system constitutes of one microcontroller. The ultrasonic sensor system mounted in the front of the vehicle system sends continuous update of the distance of the vehicle ahead. The controller evaluates this data and calculates the acceleration/deceleration of the vehicle ahead. Also an accelerometer to monitor the acceleration and deceleration of his vehicle is installed [8]. Thus the data from Ultrasonic sensor and the on board accelerometer are evaluated and compared and based on this warning messages are sent to the driver. The warning messages are sent to the driver. The driver is alerted by a panel containing a string of LEDs which show different colours based on amount of deceleration required [1].

The infrared warning system mounted on the leading vehicle is responsible for alerting the microcontroller of following vehicle to warn its driver and mitigate a crash that may be due to an impending rear-end collision [7]. The desired mode of communication required is straight optical lambertian range of communication to notify only the vehicles following the leading vehicle. The Collision avoidance system receives the IR signal transmitted by the by the leading vehicle and draws proper control action based on the time to collision that it calculates and sends the data containing the value of amount of braking to be generated to the servo motors[10]. The below block diagram shown depicts the entire system with the common communication bus.

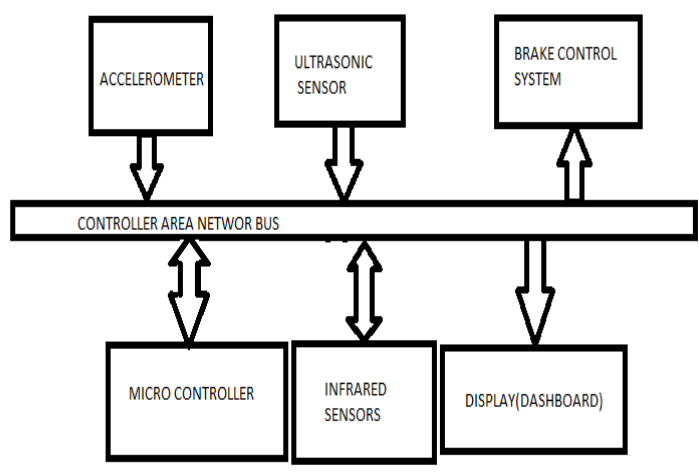

Figure 1: System Block Diagram

\section{WORK COMPLETED}

\subsection{Accelerometer}

Successful interfacing of the accelerometer sensor adxl335 has been done. Acceleration in the $\mathrm{x}, \mathrm{y}$ and $\mathrm{z}$ directions were obtained and tested. The accelerometer we have used has a range of operation from $-3 \mathrm{~g}$ to $3 \mathrm{~g}$. Since this range is sufficient for our everyday vehicles this particular sensors proves to be the most cost effective and easy to implement solution [9]. The interface circuit is as shown in figure2

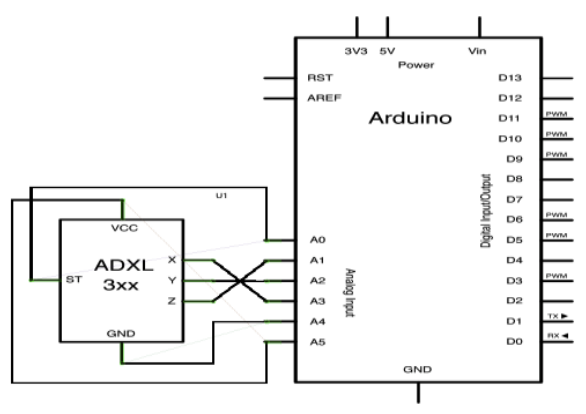

Figure 2: Accelerometer interface with Arduino UNO

\subsection{CAN Node}

A CAN Bus network has been successfully implemented wherein 2 can nodes were formed using MCP 2515 and 2551.An array of user entered data was sent to test the working of the CAN network. The accelerometer and the CAN network were also simultaneously interfaced and the readings sent over a CAN network.[12]. A CAN node was formed using one MCU and CAN controller and transceiver being interfaced on a common bus. LEDs were used to represent transmission of data. Whenever these LEDs blink, it indicated that the transmission of Data has starts. In actual practical implementation, a twisted pair cable with appropriate shielding will be used. The following figure depicts the architecture of a CAN node. 
International Journal of Computer Applications (0975 - 8887)

Volume 155 - No 9, December 2016

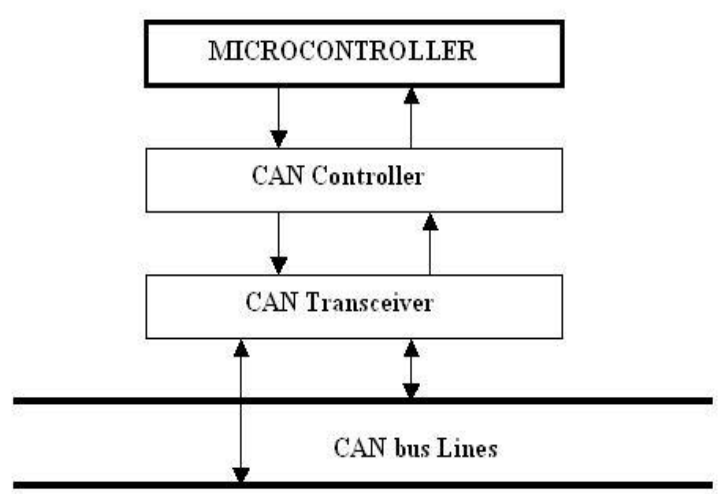

In the following images, successful transmission of data can be observed via the CAN network

Figure 3: CAN NODE

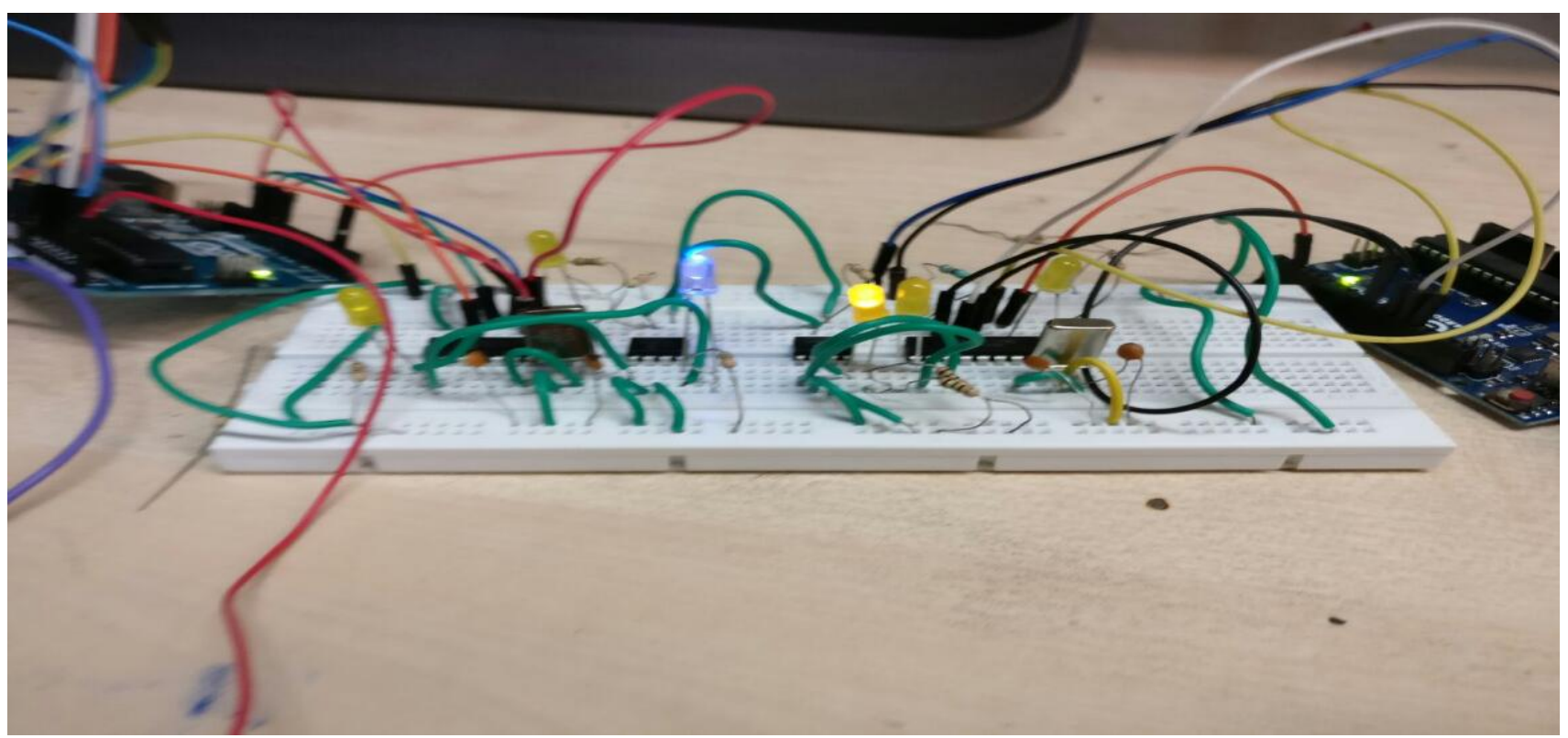

(a.)

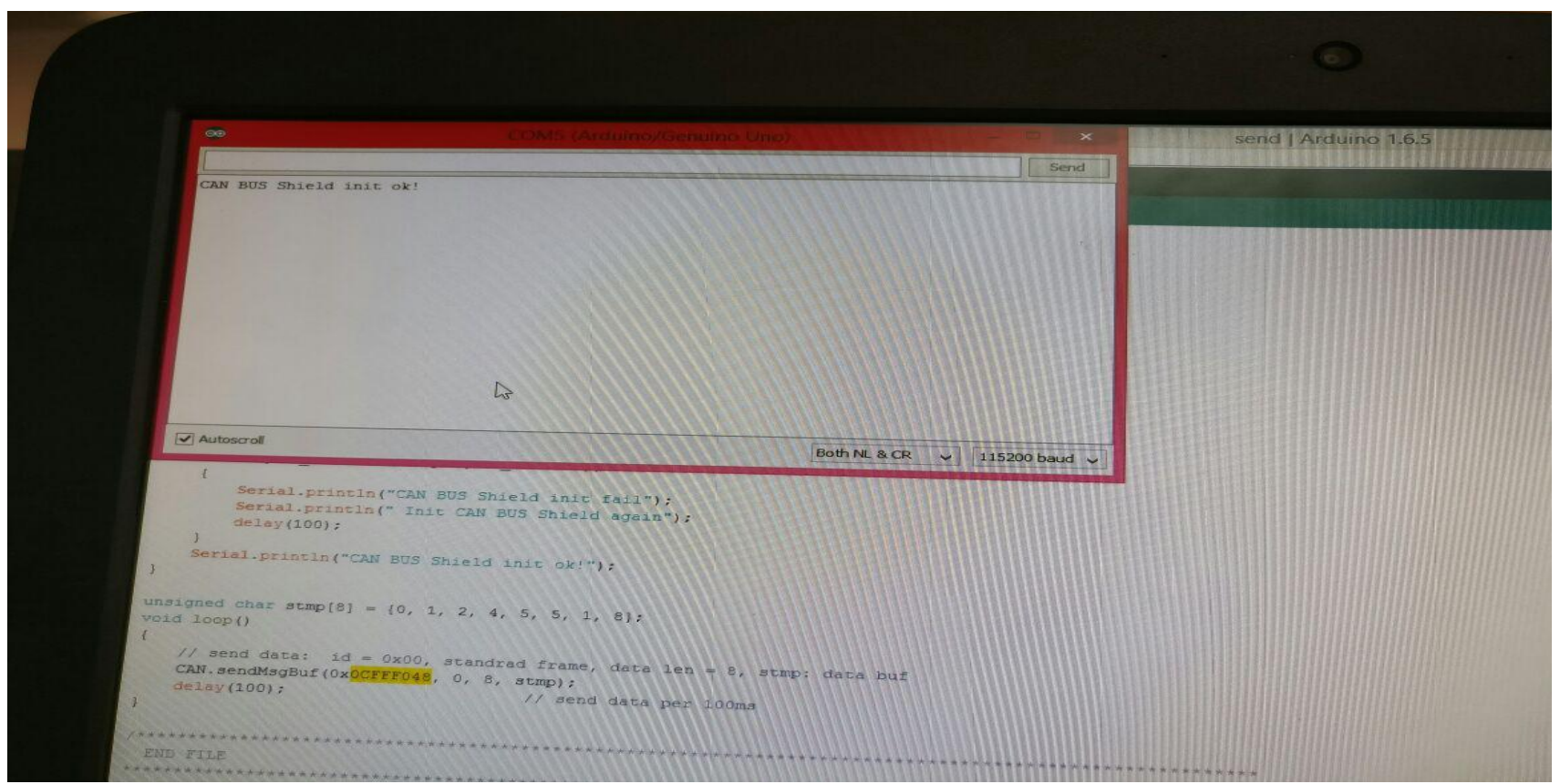

(b.) 


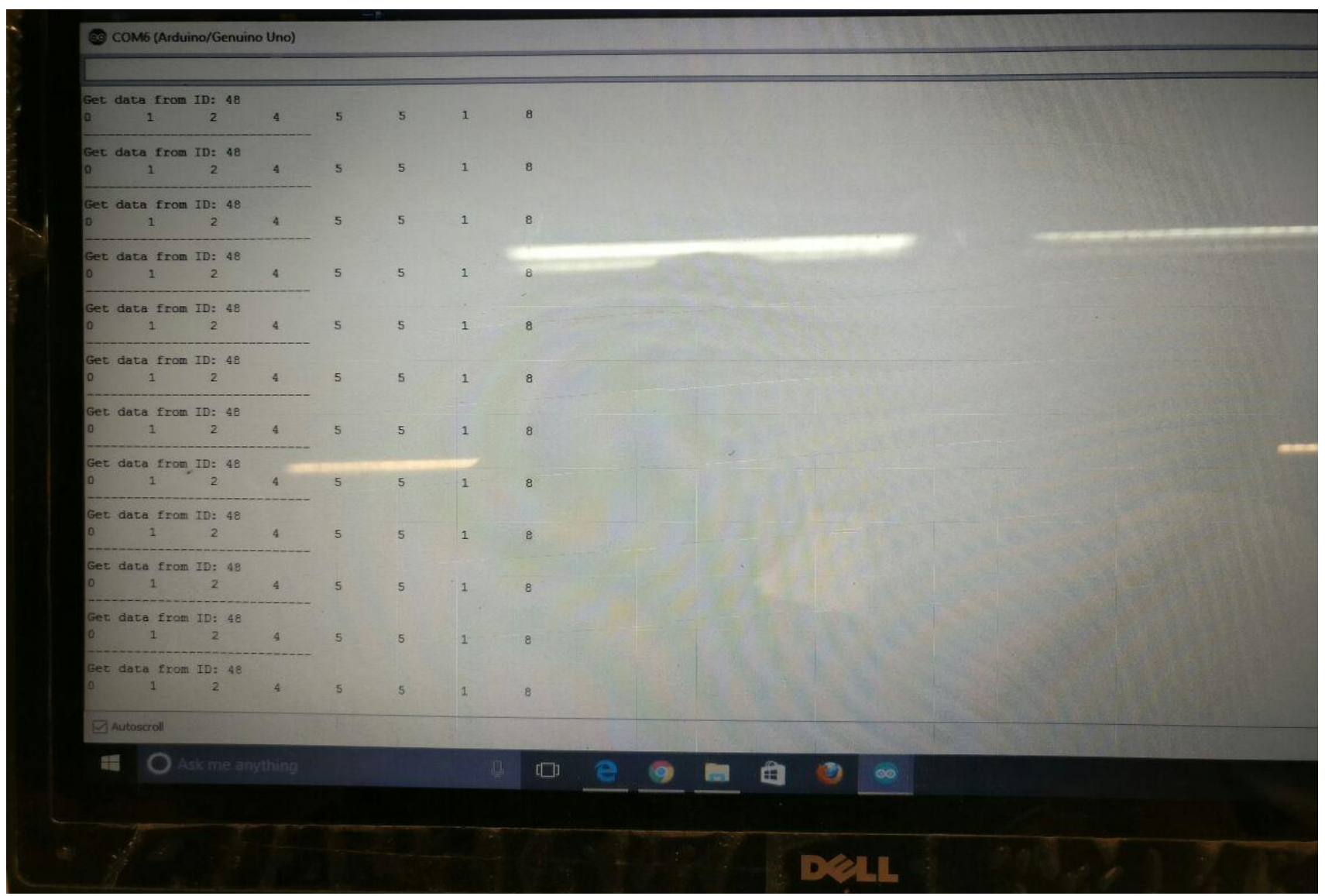

(c.)

Figure 4: (a.) CAN Bus circuit with indication LEDs (b.) CAN initialized(c.) Array of data as seen on the receiving Arduino

\subsection{Ultrasonic Sensor}

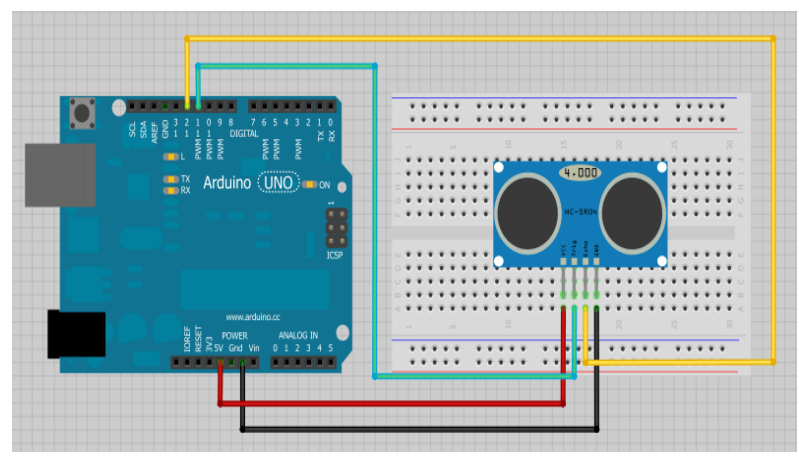

Figure 5: Ultrasonic Sensor interface with Arduino

The IR sensor was interfaced to the microcontroller and tested to work in the pre-determined ranges.

\section{FUTURE WORK}

Interfacing of all sensors to microcontroller simultaneously along with the CAN network and sending all the sensor data on the CAN bus in a real car. CAN bus to serve as a communication channel between all the systems consisting of sensors, actuators and controllers will also be set up. Packaging of braking actuators will also be decided to integrate the servo motors as well as positioning of the IR transmitter LED and their testing in real world road conditions.

\section{CONCLUSION}

The proposed system uses an Arduino UNO controller which is known for its efficient control and response time. The system is quick in response when compared with the available GPS based system in terms of response time. It is independent of any external infrastructure and network to calculate all its parameters which again increases efficiency. It is lower in cost as all its components are cheaply available. Also all the components are easy to debug and the system can easily be put up in the market. The system does not intend to notify other running vehicles except the ones that are following it or is in the lambertian line of sight of the array of IR transmitter lined in the rear bumper. These following vehicles are the most probable cause of rear end collision. Hence IR was chosen over other radiation technologies like RF, ZigBee and Bluetooth for its straight line of sight capabilities. Omnidirectional broadcasting of message was found to be undesirable in rear end collision avoidance of vehicles.

The system uses CAN for messaging which is one of the most widely used communication buses in vehicles. It is known for its robustness and low error rates.

\section{REFERENCES}

[1] "A CAN protocol based embedded system to avoid rearend collision of vehicles" Signal Processing, Informatics, Communication and Energy Systems (SPICES), 2015 IEEE International Conference on, Issue Date: 19-21 Feb. 2015, Written by: Kedareswar, P.S.; Krishnamoorthy, V 
[2] Online[online.]Available:

http://nidm.gov.in/idmc2/PDF/Presentations/road_accide nts/Pres4.pdf

[3] Online[online.].

Available:http://www.volvotrucks.com/SiteCollectionDoc uments/VTC/Corporate/Values/ART\%20Report\%202013 _150dpi.pdf

[4] Arduino website:Arduino UNO Online available: https://www.arduino.cc/

[5] Joshue Perez and Jorge Godoy 0957-4174/2012, Enrique Onieva at AUTOPIA Program at Center for Automation and Robotics-Spanish National Research Council (CARCSIC), 28500 Madrid, Spain

[6] A Collision Warning System for rear-end collision: a driving simulator study by, Francesco Bella, Roberta Russopp. 1877-0428, 2011, Elsevier Ltd.

[7] Gormer, S., Muller, D., Hold, S., Meuter, M., Kummert and A. "Vehicle recognition and ttc estimation at night based on spotlight pairing" $p p .1$
[8] Kavitha, K. V. N., Bagubali, A., Shalini and L. "V2v wireless communication protocol for rear-end collision avoidance on highways with stringent propagation delay" pp. 661-6

[9] Online[online.]. Available: http://www.analog.com/sttnatic/importedfiles/data_sheet s/ADXL335.pdf

[10] Kaempchen, N., Schiele, B., Dietmayer and K. "Situation assessment of an autonomouss emergency brake for arbitrary vehicle-to-vehicle collision scenarios" vol. 10, pp. 678-687, 2009

[11] Multiplexed Networks for Embedded Systems CAN, LIN, Flexray, Safe-by-Wire...Dominique Paret Translated by Roderick Riesco, MA Member of the Institute of Translation and Interpreting, UK2000.

[12] Ti website: http://www.ni.com/white-paper/2732/en/ 